\title{
EDITORIAL
}

\section{Elements of a flu pandemic}

\author{
M Sohail \\ Department of Biochemistry, South Parks Road, University of Oxford, Oxford OX1 3QU, England, UK. Tel: +441865 \\ 285316, Fax: +44 1865275259 (Switchboard), Email: muhammad.sohail@bioch.ox.ac.uk
}

Journal of Molecular and Genetic Medicine (2005), 1(2), 38-39

(Published 30 December 2005)

A human influenza pandemic generally occurs every 30-40 years, and many experts believe that the next pandemic is overdue. There are a number of requirements for a new pandemic to begin: i) emergence of a new viral strain to which general populations lack sufficient immunity, ii) ability of this new strain to infect humans and to cause severe disease, and, iii) capability to transmit efficiently among humans, i.e., be highly contagious. For a pandemic strain to establish at a global level it also requires a vehicle for worldwide spread.

Close similarity of reconstructed Spanish influenza pandemic virus nucleotide sequence (Tumpey et al, 2005) with that of the avian H1N1 virus (AH1N1), rather than any other mammalian HIN1 strains, and the lethality of the reconstructed virus for fertile chicken eggs, indicates that the Spanish influenza pandemic of 1917-1918 might have been started by an avian virus. This influenza pandemic killed around 50 million people globally. The large-scale spread of this influenza virus was fuelled by travelling armies of the World War 1 - the time that saw the movement of around 10 million soldiers (who also frequently carried with them live poultry as food source!).

Although no one knows which virus will be the cause of the next human influenza pandemic, one strong candidate with the capacity to set off such a pandemic is an avian influenza (bird flu) virus, AH5N1 providing one of the basic elements of an influenza pandemic. This strain belongs to the family of avian influenza retroviruses, members of which cause varying degree of respiratory disease in poultry. The strain of AH5N1 currently established in poultry is one of the most lethal known strain of this family; this strain is highly-contagious among poultry, has a relatively short incubation period (only a few hours), leading to certain death of the infected birds. The AH5N1 virus not only causes severe respiratory failure in birds, but also it causes extensive haemorrhage of internal organs - something similar to what Ebola virus does in humans.

The current outbreak of the lethal avian influenza was first detected in 1997 in Hong Kong where a large number of chickens suddenly died of severe respiratory failure and extensive haemorrhage. This region has been the source of at least one other flu pandemics in the late 1960s'. In this densely populated region general population routinely trades in live birds and animals, presenting a perfect scenario for the transmission of animal-to-human infections. Avian influenza viruses very rarely infect humans, yet the real concerns of the spread of AH5N1 to humans, possibly leading to a pandemic, cropped up when at least eighteen human cases of AH5N1 infection were detected in Hong Kong leading to six deaths. Through drastic measures, involving culling of large poultry flocks ( $\sim 1.5$ million birds) and spraying of large areas with disinfectants, this outbreak was quickly suppressed. Any further AH5N1 epidemics were unseen until its re-emergence in 2003 in poultry flocks in South Korea, Thailand and Vietnam. On this occasion this virus infected nearly 120 humans; almost half of those infected died of severe viral pneumonia and multi-organ failure. In the earlier outbreak of 1997 this virus largely infected elderly human populations; however, since the 2003 outbreak, it has infected largely younger individuals, including children. So it appears that, while it remained in dormancy for a number of years, the virus has been evolving to acquire new genetic traits. The virus also seems to be expanding its host range and has infected other animals such as, mouse model, cats and tigers (though further transmission of this virus from these animals to humans has not been reported). Worryingly, year 2005 also saw the spread of this virus through migratory birds to Russia, Turkey and Eastern Europe, and the isolation of AH5N1 from a young patient that was resistant to the common neuraminidase inhibitory anti-viral drug, osel- 
tamivir (Tamiflu) (Le et al, 2005). These events, combined with the continued common practice among general population of live bird and animal trade in Asian countries, has again fuelled the fears of the AH5N1 strain might become the cause of a human influenza pandemic.

Can AH5N1 be a cause of a flu pandemic, particularly in its current form? This virus already has the ability to cross species barrier, and it can infect and cause severe disease in humans. What it lacks is the efficient human-to-human transmissibility. Fortunately, so far this has been rare. To-date there have been only two such reported cases in Vietnam where individuals became infected with this virus, apparently without direct contact with live infected birds, leading to one death. While on one hand this shows that the general flora of this virus has limited genetic information for human-to-human transmission to cause a pandemic, on the other it is making progress in this direction.

By virtue of having an RNA genome, retroviruses have the capacity to mutate at a rapid pace, giving rise to variants with new antigenic properties. Since avian viruses are not normal human flora, the general human populations lack immunity against them. Thus for this reason, if this virus somehow acquires the capability of efficient human-to-human transmission, it already has the necessary genetic footprint to causes widespread human disease, without the need for a major antigenic shift. However, for this virus to cause a major human pandemic it needs to be highlycontagious, and ideally transmissible via droplet nuclei. The possibility is that AH5N1 can become highly-contagious among human populations either by mutating or via a genetic recombination event (similar to human-swine recombinants (e.g., Ghedin et al, 2005)) with a normal human influenza virus. However, all indications from the nucleotide sequence analysis of reconstructed Spanish influenza pandemic virus are that the avian influenza virus that might have caused the 1918 pandemic became contagious in humans without a genetic recombination event. This suggests that, in theory, it is possible for AH5N1 to become an efficient human pathogen through genetic mutations, with continuous exposure to humans.

Furthermore, by virtue of the avian virus having distinct antigenic properties not known to human hosts, it has the capacity to cause an influenza pandemic, running in parallel with another influenza pandemic caused by a different virus.

While air travel is likely to be a major fuel for the global spread of an influenza virus strain, the emer- gence of viral strains that have the ability to infect both humans and migratory birds can be even more alarming. However, generally as bacterial or viral strains evolve to become more infectious to get a strong foothold in host populations, they also tend to become less virulent. Thus, we can hope that by the time the avian virus has learned high-infectivity and contagiousness in humans, it also becomes less virulent, and that the world's health services are better equipped to handle a pandemic.

\section{REFERENCES}

Ghedin E et al. 2005. Nature, 437, 1162-1166.

Le QM et al. 2005. Nature, 437, 1108.

Tumpey TM et al. 2005. Science, 310, 77-80.

\section{SHORT COPYRIGHT STATEMENT}

This is an open access article, published under the terms of the Licence for Users available at http://www.libpubmedia.co.uk/ MedJ/LicenceForUsers.pdf. This licence permits noncommercial use, distribution and reproduction of the article, provided the original work is appropriately acknowledged with correct citation details. 